\title{
Markers of autophagy are adapted to hyperglycaemia in skeletal muscle in type 2 diabetes
}

\author{
Rikke Kruse $^{1,2}$ • Birgitte F. Vind ${ }^{2}$ - Stine J. Petersson ${ }^{1,2}$ • Jonas M. Kristensen ${ }^{1,2}$. \\ Kurt Højlund ${ }^{1,2}$
}

Received: 21 December 2014 / Accepted: 15 May 2015 /Published online: 7 June 2015

(C) Springer-Verlag Berlin Heidelberg 2015

\begin{abstract}
Aims/hypothesis Autophagy is a catabolic process that maintains cellular homeostasis by degradation of protein aggregates and selective removal of damaged organelles, e.g. mitochondria (mitophagy). Insulin resistance in skeletal muscle has been linked to mitochondrial dysfunction and altered protein metabolism. Here, we investigated whether abnormalities in autophagy are present in human muscle in obesity and type 2 diabetes.

Methods Using a case-control design, skeletal muscle biopsies obtained in the basal and insulin-stimulated states from patients with type 2 diabetes during both euglycaemia and hyperglycaemia, and from glucose-tolerant lean and obese individuals during euglycaemia, were used for analysis of mRNA levels, protein abundance and phosphorylation of autophagy-related proteins.

Results Muscle transcript levels of autophagy-related genes (ULK1, BECN1, PIK3C3, ATG5, ATG 7, ATG12, GABARAPL1, MAP1LC3B, SQSTM1, TP53INP2 and FOXO3A [also known as $F O X O 3]$ ), including some specific for mitophagy (BNIP3, BNIP3L and MUL1), and protein abundance of autophagy-related gene (ATG)7 and Bcl-2/
\end{abstract}

Electronic supplementary material The online version of this article (doi:10.1007/s00125-015-3654-0) contains peer-reviewed but unedited supplementary material, which is available to authorised users.

Kurt Højlund

kurt.hoejlund@rsyd.dk

1 The Section of Molecular Diabetes \& Metabolism, Institute of Clinical Research and Institute of Molecular Medicine, University of Southern Denmark, Odense, Denmark

2 Department of Endocrinology, Odense University Hospital, Kløvervænget 6, DK-5000 Odense, Denmark adenovirus E1B 19-kDa-interacting protein 3 (BNIP3), as well as content and phosphorylation of forkhead box O3A (FOXO3A) were similar among the groups. Insulin reduced lipidation of microtubule-associated protein light chain 3 (LC3)B-I to LC3B-II, a marker of autophagosome formation, with no effect on p62/sequestosome 1 (SQSTM1) content in muscle of lean and obese individuals. In diabetic patients, insulin action on LC3B was absent and p62/SQSTM1 content increased when studied under euglycaemia, whereas the responses of LC3B and p62/SQSTM1 to insulin were normalised during hyperglycaemia.

Conclusions/interpretation Our results demonstrate that the levels of autophagy-related genes and proteins in muscle are normal in obesity and type 2 diabetes. This suggests that muscle autophagy in type 2 diabetes has adapted to hyperglycaemia, which may contribute to preserve muscle mass.

Keywords Autophagy $\cdot$ Human $\cdot$ Hyperglycaemia $\cdot$ Skeletal muscle $\cdot$ Type 2 diabetes

$\begin{array}{ll}\text { Abbreviations } \\ \text { ATG } & \text { Autophagy-related gene } \\ \text { BNIP3 } & \begin{array}{l}\text { Bcl-2/adenovirus E1B 19-kDa-interacting } \\ \text { protein 3 }\end{array} \\ \text { BNIP3L } & \text { BNIP3-like } \\ \text { ERK } & \text { Extracellular signal-regulated kinase } \\ \text { FOXO3 } & \text { Forkhead box O3 } \\ \text { GDR } & \text { Glucose disposal rate } \\ \text { JNK } & \text { c-Jun N-terminal kinase } \\ \text { LC3 } & \text { Microtubule-associated protein light chain 3 } \\ \text { mTOR } & \text { Mechanistic target of rapamycin } \\ \text { mTORC1 } & \text { mTOR complex 1 } \\ \text { p70S6K } & \text { p70 S6 kinase }\end{array}$


qPCR Quantitative PCR

ROS Reactive oxygen species

SQSTM Sequestosome 1

TP53INP2 Tumour protein p53 inducible nuclear protein 2

\section{Introduction}

Skeletal muscle is the major site of insulin-stimulated glucose uptake $[1,2]$ and correspondingly an important site of insulin resistance in obesity and type 2 diabetes. Insulin resistance in human skeletal muscle is characterised by decreased insulinstimulated glucose disposal and metabolism, triacylglycerol accumulation, as well as reduced content and functional capacity of mitochondria [3-7]. At the molecular level, there is evidence of impaired insulin signalling through IRS-1, phosphatidylinositol 3-kinase (PI3K), Akt, and glycogen synthase in skeletal muscle of insulin-resistant individuals [8-13]. Moreover, insulin resistance in obesity and type 2 diabetes is characterised by abnormal protein metabolism $[14,15]$. The two main proteolytic systems in skeletal muscle are the ubiquitin-proteasome system and macroautophagy (hereafter referred to as autophagy). Increased content of several proteasome subunits was recently demonstrated in skeletal muscle in obesity and type 2 diabetes [16], whereas a potential role of muscle autophagy in these conditions remains to be clarified.

Autophagy is a highly conserved catabolic pathway, which is essential for the maintenance of cellular homeostasis. This process is responsible for degradation of protein aggregates and dysfunctional organelles, and is constantly ongoing at low levels in most cell types. Autophagy is therefore of particular importance in long-lived post-mitotic cells, such as skeletal muscle fibres and neurons [17]. In skeletal muscle, autophagy is known to be induced by fasting, atrophy, and exercise [18-20]. When autophagy is activated, the Unc-51 like autophagy activating kinase 1 (ULK1)-complex initiates the formation of the autophagosome [21, 22]. Recruitment of the BCL2 interaction myosin/moesin like coiled-coil protein 1 (BECLIN1)-vacuolar protein sorting 34 (VPS34) complex for generation of phosphatidylinositol 3-phosphate, as well as conjugation of autophagy-related gene (ATG)12 and ATG5 by ATG7 and ATG10, and lipidation of microtubuleassociated protein light chain 3 (LC3)-I to LC3-II, or other members of the ATG8 family (e.g. gamma-aminobutyric acid (A) receptor-associated protein like 1 [GABARAPL1]), by ATG3 and ATG7, is necessary for the formation of the mature autophagosome [21-23]. The autophagosome fuses with a lysosome for degradation of the sequestered materials [22, 24]. Autophagy was long considered a non-selective degradation pathway, but there is increasing evidence for selective forms of autophagy mediated by specific autophagy receptors, such as p62/sequestosome 1 (SQSTM1) (hereafter p62) and neighbour of BRCA1 gene 1 (NBR1) for degradation of ubiquitinated protein aggregates, and $\mathrm{Bcl}-2$ /adenovirus E1B 19-kDa-interacting protein 3 (BNIP3) and BNIP3-like (BNIP3L) for selective removal of mitochondria (mitophagy) [17, 22].

Recent reports have highlighted the importance of autophagy in skeletal muscle. Thus, muscle-specific knockout of the crucial autophagy gene, $\operatorname{Atg} 7$, in mice caused loss of muscle with accumulation of abnormal mitochondria [25, 26], decreased respiratory capacity [26] and increased oxidative stress $[25,26]$. While starvation is thought to induce autophagy in skeletal muscle, insulin may inhibit autophagy by two Akt-dependent mechanisms involving mechanistic target of rapamycin (mTOR)-mediated repression of autophagy [23, 27] and blocking of forkhead box O3 (FOXO3)-mediated transcription of autophagy-related genes such as VSP34, LC3, GABARAPL1, ATG7, BNIP3, BNIP3L, MUL1 and CTSL1 [18, 20, 28]. Since both deficient and excessive autophagy is harmful, a well-orchestrated regulation of this process is of great importance for muscle homeostasis. Based on the presence of impaired insulin signalling to Akt $[4,11]$, mitochondrial dysfunction and altered protein metabolism, we hypothesised that autophagy is dysregulated in human skeletal muscle in obesity and type 2 diabetes.

The objectives of this study were therefore to investigate whether the basal levels of autophagic markers and the activity of the autophagic machinery are altered in skeletal muscle and adipose tissue in obesity and type 2 diabetes, and whether autophagy responds differently to insulin in skeletal muscle of insulin-resistant individuals.

\section{Methods}

Study participants The present study investigated material previously collected and reported by Vind et al [4]. Compared with the original report, two additional lean individuals were included. In a case-control design, ten obese patients with type 2 diabetes were carefully matched to 12 healthy, lean and ten obese, non-diabetic volunteers (Table 1). The eligibility criteria are given in the Electronic Supplementary Material (ESM) Methods. Informed consent was obtained from all participants before participation. The study was approved by the Regional Scientific Ethical Committees for Southern Denmark and was performed in accordance with the Helsinki Declaration.

Study design Participants were instructed to abstain from strenuous physical activity for a period of $48 \mathrm{~h}$ prior to the experiment. Following an overnight fast, the lean and obese individuals underwent a euglycaemic-hyperinsulinaemic clamp with tracer glucose and combined with indirect calorimetry. Patients with type 2 diabetes were clamped twice, 
Table 1 Clinical and metabolic characteristics

\begin{tabular}{|c|c|c|c|}
\hline & Lean & Obese & Type 2 diabetic \\
\hline$n$ (female/male) & $12(6 / 6)$ & $10(4 / 6)$ & $10(6 / 4)$ \\
\hline Age (years) & $54.5 \pm 1.3$ & $55.4 \pm 1.2$ & $53.9 \pm 1.6$ \\
\hline BMI $\left(\mathrm{kg} / \mathrm{m}^{2}\right)$ & $23.4 \pm 0.5$ & $31.1 \pm 0.9^{* * *}$ & $29.8 \pm 1.3 * * *$ \\
\hline Fat mass (kg) & $17.6 \pm 3.3$ & $30.3 \pm 1.9 * *$ & $29.6 \pm 1.3 * *$ \\
\hline $\begin{array}{l}\text { Fasting plasma glucose } \\
(\mathrm{mmol} / \mathrm{l})\end{array}$ & $5.5 \pm 0.1$ & $5.8 \pm 0.1$ & $8.9 \pm 0.7 * * * \dagger$ \\
\hline $\begin{array}{l}\text { Fasting serum insulin } \\
\quad(\mathrm{pmol} / \mathrm{l})\end{array}$ & $30 \pm 4$ & $40 \pm 6$ & $85 \pm 16.3^{* * \dagger}$ \\
\hline Fasting C-peptide (pmol/l) & $507 \pm 29$ & $633 \pm 48$ & $1,181 \pm 140 * * * \dagger \dagger$ \\
\hline $\mathrm{HbA}_{1 \mathrm{c}}(\%)$ & $5.5 \pm 0.1$ & $5.3 \pm 0.1$ & $6.8 \pm 0.4^{* * * \dagger \dagger \dagger}$ \\
\hline $\mathrm{HbA}_{1 \mathrm{c}}(\mathrm{mmol} / \mathrm{mol})$ & $36.2 \pm 1.0$ & $34.3 \pm 1.3$ & $51.2 \pm 3.9 * * * \dagger \dagger$ \\
\hline $\begin{array}{l}\text { Plasma triacylglycerols } \\
(\mathrm{mmol} / \mathrm{l})\end{array}$ & $0.8 \pm 0.1$ & $1.0 \pm 0.3$ & $1.6 \pm 0.2 * * * \dagger \dagger$ \\
\hline $\begin{array}{l}\text { Serum insulin, clamp } \\
(\mathrm{pmol} / \mathrm{l})\end{array}$ & $409 \pm 19$ & $397 \pm 16$ & $428 \pm 14$ \\
\hline $\begin{array}{l}\text { GDR, basal } \\
\qquad\left(\mathrm{mg} \mathrm{min} \min ^{-1} \mathrm{~m}^{-2}\right)\end{array}$ & $76 \pm 3$ & $74 \pm 6$ & $73 \pm 3$ \\
\hline $\begin{array}{l}\text { GDR, clamp } \\
\quad\left(\mathrm{mg} \mathrm{min} \min ^{-1} \mathrm{~m}^{-2}\right)\end{array}$ & $380 \pm 25$ & $309 \pm 24^{*}$ & $160 \pm 26^{* * * i \dagger \dagger}$ \\
\hline
\end{tabular}

The data are presented as means \pm SEM

${ }^{*} p<0.05, * * p<0.01$ and $* * * p<0.001$ vs lean controls; ${ }^{\dagger} p<0.05$, ${ }^{\dagger \dagger} p<0.01$ and ${ }^{\dagger \dagger} p<0.001$ vs obese controls

separated by $4-6$ weeks, randomly starting with either a euglycaemic- or isoglycaemic-hyperinsulinaemic clamp as described [4]. All participants were asked to eat the same meal on the evening before the study.

Euglycaemic/isoglycaemic-hyperinsulinaemic clamp and muscle biopsies The study participants initially underwent a $2 \mathrm{~h}$ basal tracer $\left(3-\mathrm{H}^{3}\right.$-glucose) equilibration period after which a euglycaemic-hyperinsulinaemic clamp $(4 \mathrm{~h}$ insulin infusion using $40 \mathrm{mU} \mathrm{m}{ }^{-2} \mathrm{~min}^{-1}$ ) was performed [9, $10,29]$. Muscle biopsies were taken from $m$. vastus lateralis before and after the $4 \mathrm{~h}$ insulin infusion period. Details about the clamp and biopsies are given in ESM Methods.

RNA isolation and cDNA synthesis For isolation of total RNA, 10-20 mg muscle biopsy and 30-70 mg subcutaneous fat biopsy were homogenised in $1 \mathrm{ml}$ TRI reagent (SigmaAldrich, St Louis, MO, USA) with ceramic beads (1.4 mm zirconium oxide beads) using the PreCellys 24 system (5, $000 \mathrm{rpm} / 2 \times 20 \mathrm{~s}$; Bertin Technologies, Montigny-LeBretonneux, France). Hereafter, total RNA was extracted according to the manufacturer's guidelines. The RNA concentration was determined using a NanoDrop 1000 (Thermo Scientific, Waltham, MA, USA) and $1.5 \mu \mathrm{g}$ total RNA was subsequently treated with DNaseI (Amplification Grade, Invitrogen, Carlsbad, CA, USA) according to the manufacturer's instructions and reverse transcribed using a high capacity cDNA reverse transcription kit (Applied Biosystems, Foster City, CA, USA).
Quantitative real-time PCR analysis Real-time quantitative PCR (qPCR) was performed on an ABI Prism 7900HT Sequence Detection system (Applied Biosystems) using TaqMan custom Arrays (Applied Biosystems) according to the manufacturer's instructions. The specific primer-probe pairs used are listed in ESM Table 1. All samples were run in triplicate and the data analysed using qBasePlus Biogazelle software (Zwijnaarde, Belgium) [30, 31]. In both adipose tissue and skeletal muscle, the mRNA levels were normalised to the geometric mean of two suitable reference genes, which were not different among the groups and were not affected by insulin. The mRNA levels in muscle were normalised to PPIA and B2M, while mRNA levels in adipose tissue were normalised to PPIA and $A C T B$.

Muscle lysates, SDS-PAGE and western blot For extraction of protein, frozen muscle biopsies were freeze-dried, dissected free of visible fat, blood and connective tissue. The muscle lysates were prepared as described in ESM Methods. Muscle proteins were separated by SDS-PAGE on self-cast $8-15 \%$ Tris-HCl polyacrylamide gels, and transferred (semidry) to a PVDF membrane (Immobilon transfer membrane, Millipore, Denmark). For each protein probed on a western blot, the same amount of total lysate protein was loaded for each sample on the gels. Western blots were conducted in a balanced design (samples from all experimental conditions present on all gels) and internal control samples were included on each gel to correct for possible variations between gels. On one gel, a standard curve of muscle lysate was loaded to ensure that quantification of each protein probed for was within the linear range. In the western blot analyses, the relative protein abundance from a known quantity of total lysate protein was determined. Further details and the identity and specificity of the primary antibodies used are given in ESM Methods and ESM Table 2.

Statistics The experimenters were not blinded to group assignment. The statistical analyses were performed using Sigma-Stat version 3.5 (Systat Software, San Jose, CA, USA). The data were transformed in cases where they did not display normal distribution or equal variance. Two-tailed paired $t$ tests and one-way or two-way ANOVA with repeatedmeasures using Student-Newman-Keuls post hoc testing were used to evaluate differences among and within groups. Significance was accepted at $p<0.05$. Data are presented as means \pm SEM.

\section{Results}

Clinical and metabolic characteristics At study entry, fasting levels of plasma glucose and triacylglycerols, glycated haemoglobin and serum insulin and C-peptide were elevated 
in the diabetic group compared with both control groups (Table 1). Insulin administration increased glucose disposal rate (GDR) in all three groups. However, insulin-stimulated GDR was reduced in the diabetic group compared with both control groups, but was also slightly lower in obese vs lean individuals.

Muscle and adipose tissue transcript levels of autophagyrelated genes The mRNA levels of genes encoding proteins involved in autophagy were examined by real-time qPCR. The analysis showed no differences in the muscle transcript levels of ULK1, BECN1, PIK3C3, ATG5, ATG7, ATG12, MAP1LC3B, GABARAPL1, SQSTM1, BNIP3, BNIP3L, MUL1, TP53INP2, CTSL1 and FOXO3A [also known as FOXO3] among the lean, obese and diabetic groups in the basal state (Fig. 1a-b). Furthermore, we found no differences

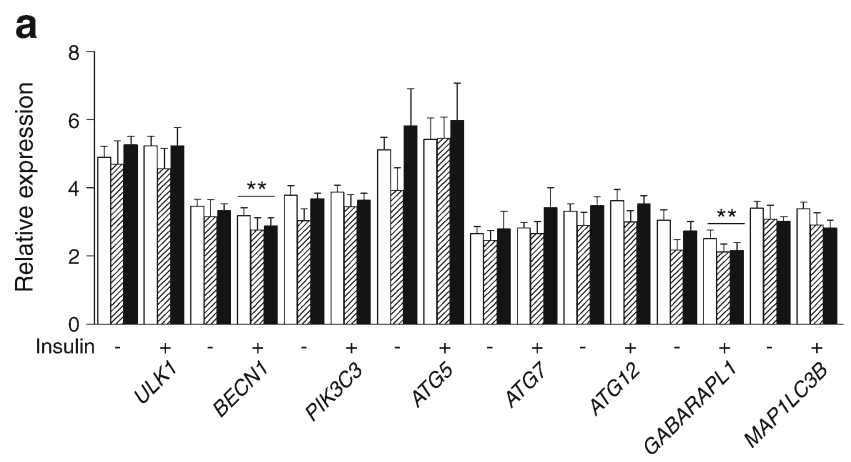

b
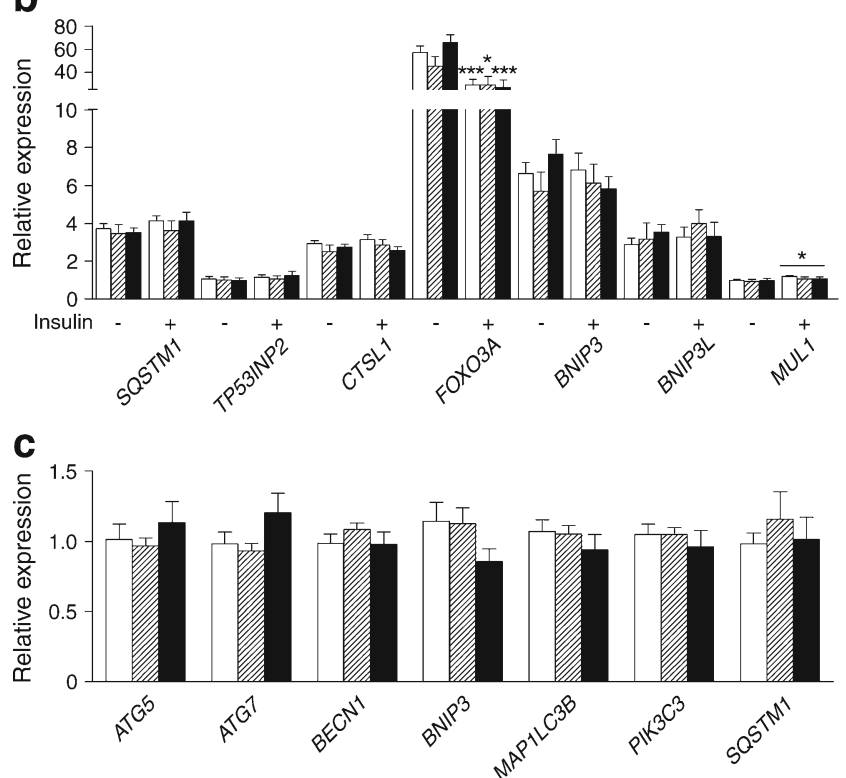

Fig. 1 mRNA expression of genes involved in autophagy in the basal and insulin-stimulated state in skeletal muscle of lean (white bars, $n=12$ ), obese (hatched bars, $n=8$ ) and type 2 diabetic (black bars, $n=10$ ) individuals $(\mathbf{a}, \mathbf{b})$, and $(\mathbf{c})$ in the basal state in subcutaneous adipose tissue (lean, $n=8$; obese, $n=8$; type 2 diabetes, $n=7$ ). The data are presented as means \pm SEM. $* p<0.05, * * p<0.01$ and $* * * p<0.001$ basal vs insulin-stimulated in the expression of $A T G 5, A T G 7, B E C N 1, B N I P 3$, $M A P 1 L C 3 B, P I K 3 C 3$ and SQSTM1 in subcutaneous adipose tissue in the basal period (Fig. 1c). Four hours of insulin infusion, which increased serum insulin to $\sim 400 \mathrm{pmol} / 1$, decreased the expression of BECN1 and GABARAPL1, while it increased MUL1 expression in skeletal muscle, with no differences among the groups (Fig. 1a-b). Furthermore, insulin decreased $F O X O 3 A$ expression in muscle from all three groups (Fig. 1b). When evaluating the fold-changes induced by insulin, no significant differences in any of the above mentioned muscle transcripts were observed among the groups (data not shown).

Abundance of key proteins involved in autophagy in skeletal muscle To identify potential regulation of autophagy at the post-transcriptional level, the content of selected key proteins involved in autophagy was investigated. No differences in the protein abundance of ATG7, BNIP3 and p62 were observed among the groups in the basal, resting state, and protein content of ATG7 and BNIP3 was not regulated by insulin (Fig. 2a-b). Previous work has shown that the level of LC3B-II is closely correlated with the number of autophagosomes, thus serving as a good indicator of autophagosome formation [32]. To elucidate whether autophagosome formation was different among the lean, obese and diabetic groups, the levels of LC3B-I and LC3B-II protein were measured and the LC3B-II/I ratio was calculated (Fig. 2c-e). This was enabled by the fact that the two LC3B isoforms migrate differently in SDS-PAGE and the shift in isoform can be detected using western blotting. Four hours of insulin infusion reduced the protein content of LC3B-II and the LC3B-II/I ratio in skeletal muscle in the lean and the obese group indicating that autophagy is inhibited by insulin administration. This effect of insulin was not seen for the LC3B-II protein content or LC3B-II/I ratio $(p=0.515$ and $p=0.111$, respectively) in muscle of diabetic patients when studied under euglycaemia. We identified no changes in the abundance of LC3B-I.

The protein levels of LC3B-II alone cannot be used as a conclusive marker of the autophagic flux [32]. We therefore determined the abundance of the autophagy receptor p62, which is degraded during autophagy in most cell types [32]. In this study, we observed no effect of insulin on the protein content of p62 in muscle of lean and obese individuals. However, p62 content was significantly increased in response to insulin in muscle of diabetic patients, when studied under euglycaemia, indicating impaired breakdown of autophagosomes (Fig. 2f).

Effect of hyperglycaemia on muscle autophagy in type 2 diabetes During the $4 \mathrm{~h}$ euglycaemic-hyperinsulinaemic clamp, the plasma glucose levels of diabetic patients were lowered from 8.9 to $5.4 \mathrm{mmol} / \mathrm{l}$. The metabolic characteristics 
a
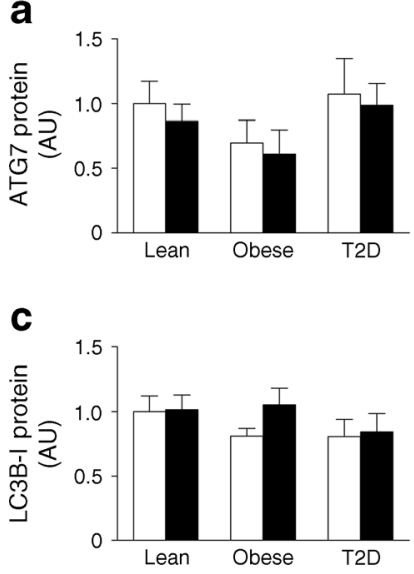

e
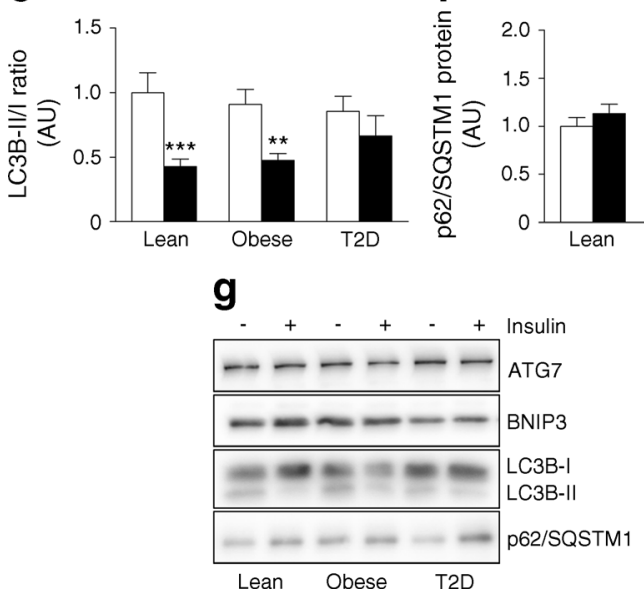

Fig. 2 Protein content of (a) ATG7, (b) BNIP3, (c) LC3B-I, (d) LC3B-II, (e) LC3B-II/I ratio and (f) p62/SQSTM1 in skeletal muscle of lean $(n=$ $12)$, obese $(n=8)$ and type 2 diabetic (T2D) individuals $(n=9)$ before (white bars) and after insulin (black bars). (g) Representative blots. Data are means \pm SEM. $\left({ }^{*}\right) p=0.075,{ }^{* *} p<0.01$ and ${ }^{* * *} p<0.001$ basal vs insulin-stimulated. AU, arbitrary units

for the isoglycaemic-hyperinsulinaemic clamp are summarised in ESM Table 3. To examine whether this decline in plasma glucose has an impact on autophagy, we compared the effect of insulin on the LC3B-II and p62 protein abundance in muscle of patients with type 2 diabetes $(n=8)$, who underwent both a euglycaemic- and an isoglycaemichyperinsulinaemic clamp (ESM Table 3). This revealed that despite an inability of insulin to decrease LC3B-II protein abundance or the LC3B-II/I ratio under euglycaemia ( $p=$ 0.365 and $p=0.083$, respectively), administration of insulin significantly decreased LC3B-II protein and the LC3B-II/I ratio during hyperglycaemia (Fig. 3a-b). Interestingly, the increase in p62 content seen upon insulin administration under euglycaemia was abolished when the patients were studied under isoglycaemic conditions (prevailing levels of fasting hyperglycaemia) ( $p=0.542$; Fig. 3c). Also, when comparing insulin-induced changes ( $\delta$-values), hyperglycaemia significantly enhanced suppression of LC3B-II protein $(p=0.024)$
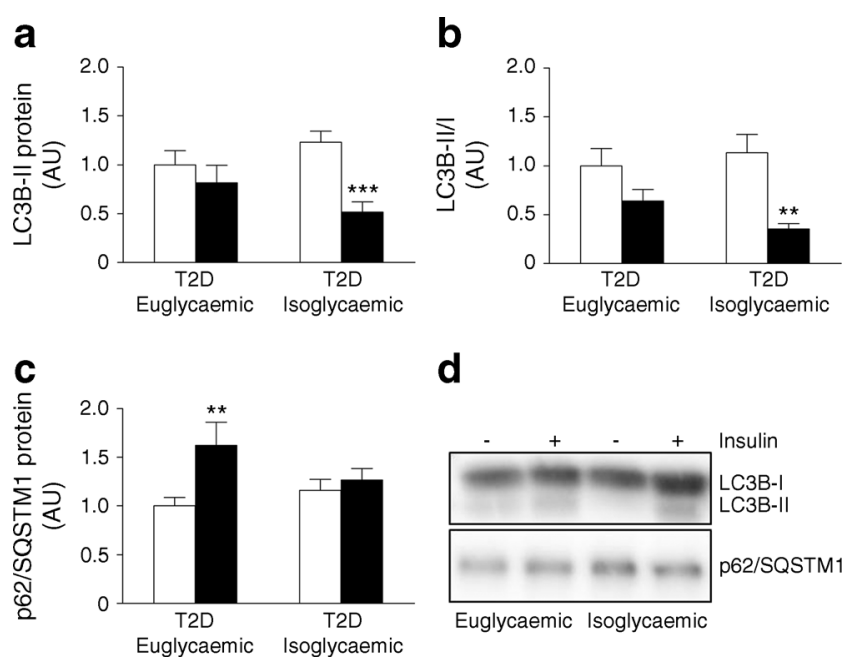

d

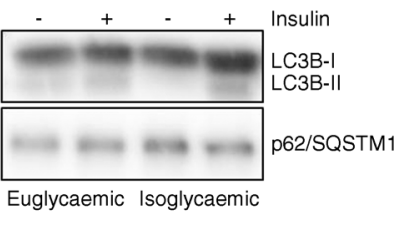

Fig. 3 Protein content of (a) LC3B-II, (b) LC3B-II/I ratio and (c) p62/ SQSTM1 in skeletal muscle of patients with type 2 diabetes (T2D) before (white bars, $n=8$ ) and after (black bars, $n=8$ ) a euglycaemic- and an isoglycaemic-hyperinsulinaemic clamp, respectively. (d) Representative blots. Data are means \pm SEM. ${ }^{* *} p<0.01$ and $* * * p<0.001$ basal vs insulin-stimulated. AU, arbitrary units

and the LC3B-II/I ratio $(p=0.047)$, and tended to attenuate $(p=0.073)$ the increase in $\mathrm{p} 62$ observed during euglycaemia.

No apparent differences in the activity of FOXO3A and mTOR To identify whether known autophagic regulators, such as the mTOR kinase and the transcription factor FOXO3A, mediate the inhibitory effect of insulin on autophagy in human skeletal muscle, we measured the protein abundance and phosphorylation of FOXO3A and the p70 S6 kinase (p70S6K), a downstream mTOR target. Insulin significantly upregulated FOXO3A protein content (Fig. 4a), whereas no changes either in the Ser 253 phosphorylation of FOXO3A (Fig. 4c) or in the FOXO3A pSer253/protein ratio (data not shown) were seen (see also ESM Fig. 1). Insulin increased Thr389 phosphorylation of p70S6K (Fig. 4d), but there were no differences in the p70S6K protein content (Fig. 4b) or in the p70S6K pThr389/protein ratio (data not shown). We did not identify any differences among the groups when we measured the p70 S6K and FOXO3A phosphorylation and protein abundance.

\section{Discussion}

In this study, we examined several key markers of autophagy in the basal, resting state in skeletal muscle and adipose tissue, as well as the autophagic response to insulin in skeletal muscle of patients with type 2 diabetes and lean and obese, glucosetolerant individuals. Overall, we found no influence of obesity or type 2 diabetes on basal mRNA levels or protein abundance of autophagic markers in skeletal muscle or fat, despite hyperglycaemia in patients with type 2 diabetes. 

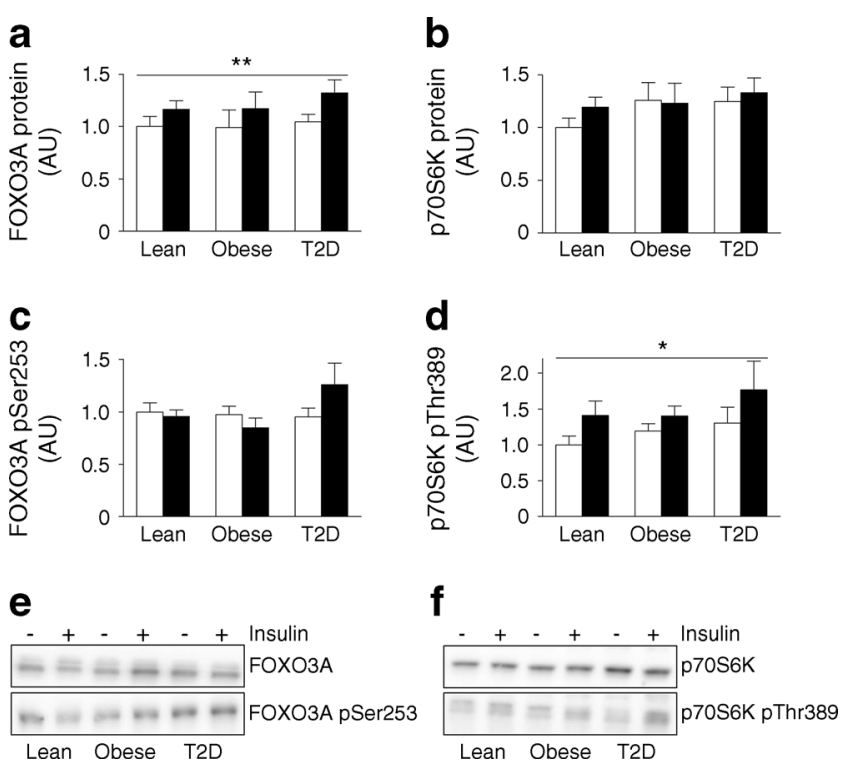

Fig. 4 Protein abundance of (a) FOXO3A and (b) S6K, and (c) Ser253 phosphorylation (pSer253) of FOXO3A and (d) Thr389 phosphorylation (pThr389) of S6K in skeletal muscle of lean $(n=12)$, obese $(n=8)$ and type 2 diabetic (T2D) individuals $(n=9)$ before (white bars) and after insulin (black bars). (e, f) Representative blots. Data are means \pm SEM. ${ }^{*} p<0.05$ and ${ }^{*} p<0.01$ basal vs insulin-stimulated. AU, arbitrary units

Furthermore, we show that stimulation with physiological insulin concentrations decreases the protein content of LC3B-II, a key marker of autophagosome formation, in skeletal muscle of lean and obese individuals. Interestingly, this response was absent in diabetic patients when studied during euglycaemia, but normalised when studied at their prevailing levels of fasting hyperglycaemia. Our results provide evidence that markers of autophagy in skeletal muscle and fat are not affected by obesity and type 2 diabetes, and suggest that muscle autophagy has adapted to hyperglycaemia in type 2 diabetes. This adaptation may in part contribute to the relative preservation of muscle mass seen in type 2 diabetes as compared with the muscle loss seen in dysregulated patients with type 1 diabetes.

Little is known about the activity and regulation of autophagy in humans, but there is increasing evidence to support the hypothesis of dysregulated autophagy in obesity and type 2 diabetes. First, impaired insulin signalling, mitochondrial dysfunction and altered protein metabolism in skeletal muscle and/or adipose tissue are known characteristics of insulin resistance and type 2 diabetes [3, 5-9, 11-13], which may all relate to perturbations in autophagy, including selective degradation of mitochondria (mitophagy) $[25,26]$. Second, knockout of $\operatorname{Atg} 7$ in skeletal muscle of mice leads to loss of muscle mass, abnormal mitochondria, decreased respiratory capacity and increased production of reactive oxygen species (ROS) $[25,26]$. This suggests that impaired autophagy produces abnormalities similar to those reported in muscle of patients with type 2 diabetes. Furthermore, there is experimental evidence that hyperglycaemia can cause both inhibition and induction of autophagy [33]. One mechanism by which hyperglycaemia may induce autophagy seems to involve activation of $\mathrm{p} 38$ mitogen-activated protein (MAP)kinase, extracellular signal-regulated kinase (ERK) or c-Jun $\mathrm{N}$-terminal kinase (JNK) due to increased oxidative stress and ROS production [33]. Consistently, hyperglycaemiaassociated oxidative stress was recently shown to induce autophagy through induction of the ROS-ERK/JNK-p53 pathway in skeletal muscle of Goto Kakizaki rats [34]. A major finding in the present study is that the basal levels of multiple markers of autophagy in skeletal muscle and fat of patients with type 2 diabetes are not different from the levels seen in lean and obese individuals, despite hyperglycaemia. Together with the observation that hyperglycaemia normalised insulin action on markers of autophagosome formation in muscle of diabetic patients, our data indicate that muscle autophagy has adapted to hyperglycaemia in type 2 diabetes. This adaptation to hyperglycaemia in one of the main proteolytic systems may, in part, explain why muscle mass is relatively spared in type 2 diabetes.

In obesity, chronic exposure to an increased calorie intake may negatively affect autophagy in major metabolic tissues such as liver, skeletal muscle and adipose tissue. Indeed, a decrease in hepatic autophagy has been reported in both genetic and dietary-induced mouse models of insulin resistance and obesity [35-38]. To our knowledge, the influence of obesity on autophagy in human skeletal muscle has not previously been reported. In the present study, we show that basal and insulin-stimulated mRNA levels and protein abundance of autophagic markers are similar in skeletal muscle of lean and obese individuals. Moreover, the insulin-mediated inhibition of LC3B lipidation in skeletal muscle was intact in obesity. These data suggest that obesity per se, does not influence autophagy in human skeletal muscle. Since the insulin sensitivity in the obese individuals was only slightly lower than in the lean individuals, further studies are needed to rule out that a higher degree of insulin resistance or morbid obesity in the absence of hyperglycaemia may affect autophagy in skeletal muscle.

There is increasing evidence that insulin resistance in skeletal muscle in obesity and type 2 diabetes is linked to abnormalities in content, function and morphology of mitochondria $[6,7,16,39,40]$. These reports suggest a potential role for altered muscle mitophagy in obesity and type 2 diabetes. BNIP3 and BNIP3L act as mitophagy receptors that target mitochondria for removal by autophagosomes [17, 21], whereas mitochondrial E3 ubiquitin protein ligase 1 (MUL1) is a mitochondrial E3 ligase, which has been shown to promote the fragmentation and clearance of mitochondria through mitophagy during skeletal muscle wasting [28]. The fact that we did not identify any differences in the expression of BNIP3, BNIP3L and MUL1 or protein abundance of BNIP3 
among the groups suggests that mitophagy is not influenced by obesity and has adapted to hyperglycaemia in type 2 diabetes. We observed a small but significant increase in MUL1 expression after insulin stimulation with no differences among the groups. This suggests the possibility that insulin promotes clearance of damaged mitochondria. However, given that insulin is known to cause an overall inhibition of autophagy, further studies are warranted to confirm a potential positive effect of insulin on mitophagy.

The insulin-mediated decrease in the lipidation of LC3B, a marker of autophagosome formation, provides evidence that autophagy is inhibited in response to insulin in skeletal muscle of lean and obese, glucose-tolerant individuals. In most cell types, induction of autophagic flux is reflected by both increased levels of LC3B-II and reduced levels of p62 [41]. However, in some cell types, there is no change in p62 levels despite strong levels of autophagy induction [41]. In the present study, we observed no effect of insulin on p62 abundance in skeletal muscle from either lean or obese individuals. This is consistent with recent data showing no effect of insulin on p62 levels in muscle biopsies from young healthy male individuals before or after prolonged fasting [42]. These data suggest that p62 is not a useful marker of insulin-mediated inhibition of autophagy in human skeletal muscle.

While starvation is known to be a potent inducer of autophagy [43], a similar role of glucose deprivation is less well established in mammals [33]. However, the lack of insulinmediated suppression of muscle LC3B-II in type 2 diabetes, when studied under euglycaemia, suggests the possibility that the inhibitory action of insulin on LC3B lipidation was counteracted by a simultaneous positive effect of glucoselowering on autophagosome formation. If the inhibitory action of insulin on autophagy is independent of p62 content in skeletal muscle, as suggested by our data and a recent report [42], then glucose-lowering may increase autophagolysosomal degradation of another cargo receptor causing p62 to accumulate by displacement with no net effect on LCB3 lipidation. Such a p62-independent mechanism involving tumour protein p53 inducible nuclear protein 2 (TP53INP2) was proposed recently [44]. In fact, we observed increased p62 content in patients with type 2 diabetes in response to insulin when studied at euglycaemia. To test whether this increase in p62 was explained by displacement, we measured TP53INP2 expression in the muscle samples. Although we could not prove a significant insulin-mediated increase in TP53INP2, the muscle transcript levels of TP53INP2 tended to increase in response to insulin in diabetic patients studied at euglycaemia. In contrast to a recent report of decreased muscle expression of TP53INP 2 in obesity and type 2 diabetes [44], we could not demonstrate any differences in TP53INP2 levels among lean, obese and diabetic individuals. This is consistent with the similar basal levels of all autophagic markers including p62. In the study of Sala et al [44], the average age in the obese and diabetic groups was much higher than in the lean group, suggesting that this was responsible for reduced TP53INP2 [44]. Overall, our results do not support the hypothesis that TP53INP2 displaces p62 as a cargo receptor as proposed recently [44]. On the other hand, we are unable to provide measurements of TP53INP2 protein levels or studies of co-localisation of p62 and LC3, and of TP53INP2 and LC3, which would be necessary to clearly support or disapprove the hypothesis.

A previous study of muscle from almost the same cohort showed a robust insulin-mediated phosphorylation of Akt at Thr308 and Ser403 in all groups, although these effects were significantly reduced in patients with type 2 diabetes [4]. However, this effect of insulin on Akt was not reflected by significant changes in the activity of the mTOR target, p70S6K, or the transcription factor FOXO3A - evaluated as the phosphorylation/protein ratios - in either glucose-tolerant or diabetic individuals. The apparent absence of insulinmediated activation of the mTOR pathway has previously been reported in healthy individuals and may be due to a decrease in circulating amino acids during insulin infusion [45]. In addition to Akt-dependent phosphorylation, a Rasrelated GTP binding protein A (RAG)-dependent translocation to the lysosomal surface seems necessary for mTOR complex 1 (mTORC1) activation [46, 47]. Thus, measurement of p70S6K may not fully account for the mTORC1-mediated regulation of autophagy [20,48]. Although FOXO3 has been shown to regulate autophagy activity in skeletal muscle $[18$, 20], the lack of changes in FOXO3A phosphorylation in our study is consistent with the absence of transcriptional regulation of most autophagy-related genes after insulin stimulation. Taken together, our data suggest that insulin activation of Akt in skeletal muscle of lean and obese individuals is not associated with measureable changes in the mTOR target $\mathrm{p} 70 \mathrm{~S} 6 \mathrm{~K}$ or FOXO3A activity, which could explain the observed reduction in LC3B-II. Furthermore, the reduced insulin action on Akt observed in diabetic muscle, both at euglycaemia and hyperglycaemia [4], suggests that the normalisation of muscle autophagy by hyperglycaemia in type 2 diabetes is, at least in part, Akt-independent.

The limitations of the present study include the small sample size and the lack of sequential muscle biopsies. Thus, for some markers of autophagy, we may have insufficient power to exclude small $(<20 \%)$, but potentially relevant differences, and we have no data on the time course of autophagy regulation. Moreover, the observational nature of the data, the inability to visualise the dynamic machinery of autophagy, and to perform mechanistic studies, means that our study provides only one piece of evidence that autophagy may be normal in obesity and has adapted to hyperglycaemia in type 2 diabetes.

In summary, we have shown, for the first time, that there are no differences in the basal mRNA or protein levels of multiple markers of autophagy in skeletal muscle or 
subcutaneous adipose tissue among lean and obese, glucosetolerant individuals and patients with type 2 diabetes. Moreover, we have shown that physiological insulin concentrations decrease a marker of autophagosome formation, and that this effect does not appear to involve regulation of the mTOR kinase or FOXO3A. Furthermore, we demonstrate that the effect of insulin on markers of autophagosome formation in muscle is lost in patients with type 2 diabetes, when studied at euglycaemia, but restored when the same patients are clamped at their prevailing level of fasting hyperglycaemia. These findings suggest that autophagy in skeletal muscle of patients with type 2 diabetes has adapted to hyperglycaemia.

Acknowledgements We acknowledge L. Hansen and C. B. Olsen (Department of Endocrinology, Odense University Hospital, Odense, Denmark); A. S. Pedersen (Institute of Molecular Medicine, University of Southern Denmark, Odense, Denmark); and B. Bolmgren (Department of Nutrition, Exercise and Sports, Copenhagen University, Copenhagen, Denmark) for skilled technical assistance. Some of the data were presented as an abstract at the ADA 73rd Scientific Sessions in 2013.

Funding The study was supported by grants from the Danish Council for Independent Research (Sapere Aude, DFF Starting Grant) and from the Novo Nordisk Foundation.

Duality of interest The authors declare that there is no duality of interest associated with this manuscript.

Author contributions RK, KH, BFV, SJP and JMK contributed to the conception and design of the study, the acquisition of data, or analysis and interpretation of data. RK drafted the manuscript, and KH, BFV, SJP and JMK revised it critically for important intellectual content and approved the final version to be published. $\mathrm{KH}$ is the guarantor of this work and as such, had full access to all the data in the study and takes full responsibility for the integrity of the data and the accuracy of the data analysis.

\section{References}

1. Shulman GI, Rothman DL, Jue T, Stein P, DeFronzo RA, Shulman RG (1990) Quantitation of muscle glycogen synthesis in normal subjects and subjects with non-insulin-dependent diabetes by $13 \mathrm{C}$ nuclear magnetic resonance spectroscopy. N Engl J Med 322:223-228

2. Baron AD, Brechtel G, Wallace P, Edelman SV (1988) Rates and tissue sites of non-insulin- and insulin-mediated glucose uptake in humans. Am J Physiol 255:E769-E774

3. Petersen KF, Befroy D, Dufour S et al (2003) Mitochondrial dysfunction in the elderly: possible role in insulin resistance. Science 300:1140-1142

4. Vind BF, Birk JB, Vienberg SG et al (2012) Hyperglycaemia normalises insulin action on glucose metabolism but not the impaired activation of AKT and glycogen synthase in the skeletal muscle of patients with type 2 diabetes. Diabetologia 55:1435-1445

5. Mogensen M, Sahlin K, Fernstrom M et al (2007) Mitochondrial respiration is decreased in skeletal muscle of patients with type 2 diabetes. Diabetes 56:1592-1599

6. Phielix E, Schrauwen-Hinderling VB, Mensink M et al (2008) Lower intrinsic ADP-stimulated mitochondrial respiration underlies in vivo mitochondrial dysfunction in muscle of male type 2 diabetic patients. Diabetes 57:2943-2949
7. Kelley DE, He J, Menshikova EV, Ritov VB (2002) Dysfunction of mitochondria in human skeletal muscle in type 2 diabetes. Diabetes 51:2944-2950

8. Hojlund K, Beck-Nielsen H (2006) Impaired glycogen synthase activity and mitochondrial dysfunction in skeletal muscle: markers or mediators of insulin resistance in type 2 diabetes? Curr Diabetes Rev 2:375-395

9. Hojlund K, Staehr P, Hansen BF et al (2003) Increased phosphorylation of skeletal muscle glycogen synthase at $\mathrm{NH} 2$-terminal sites during physiological hyperinsulinemia in type 2 diabetes. Diabetes 52:1393-1402

10. Hojlund K, Birk JB, Klein DK et al (2009) Dysregulation of glycogen synthase. J Clin Endocrinol Metab 94:4547-4556

11. Vind BF, Pehmoller C, Treebak JT et al (2011) Impaired insulininduced site-specific phosphorylation of $\mathrm{TBC} 1$ domain family, member 4 (TBC1D4) in skeletal muscle of type 2 diabetes patients is restored by endurance exercise-training. Diabetologia 54:157-167

12. Zick Y (2005) Ser/Thr phosphorylation of IRS proteins: a molecular basis for insulin resistance. Sci STKE 2005:e4

13. Saltiel AR, Kahn CR (2001) Insulin signalling and the regulation of glucose and lipid metabolism. Nature 414:799-806

14. Gougeon R, Morais JA, Chevalier S, Pereira S, Lamarche M, Marliss EB (2008) Determinants of whole-body protein metabolism in subjects with and without type 2 diabetes. Diabetes Care $31: 128-133$

15. Stump CS, Short KR, Bigelow ML, Schimke JM, Nair KS (2003) Effect of insulin on human skeletal muscle mitochondrial ATP production, protein synthesis, and mRNA transcripts. Proc Natl Acad Sci U S A 100:7996-8001

16. Hwang $\mathrm{H}$, Bowen BP, Lefort $\mathrm{N}$ et al (2010) Proteomics analysis of human skeletal muscle reveals novel abnormalities in obesity and type 2 diabetes. Diabetes 59:33-42

17. Vainshtein A, Grumati P, Sandri M, Bonaldo P (2014) Skeletal muscle, autophagy, and physical activity: the menage a trois of metabolic regulation in health and disease. J Mol Med (Berl) 92: $127-137$

18. Mammucari C, Milan G, Romanello V et al (2007) FoxO3 controls autophagy in skeletal muscle in vivo. Cell Metab 6:458-471

19. Grumati P, Coletto L, Schiavinato A et al (2011) Physical exercise stimulates autophagy in normal skeletal muscles but is detrimental for collagen VI-deficient muscles. Autophagy 7:1415-1423

20. Zhao J, Brault JJ, Schild A et al (2007) FoxO3 coordinately activates protein degradation by the autophagic/lysosomal and proteasomal pathways in atrophying muscle cells. Cell Metab 6: $472-483$

21. Kroemer G, Marino G, Levine B (2010) Autophagy and the integrated stress response. Mol Cell 40:280-293

22. Mizushima N, Yoshimori T, Ohsumi Y (2011) The role of Atg proteins in autophagosome formation. Annu Rev Cell Dev Biol 27:107-132

23. Mizushima N, Klionsky DJ (2007) Protein turnover via autophagy: implications for metabolism. Annu Rev Nutr 27:19-40

24. He C, Klionsky DJ (2009) Regulation mechanisms and signaling pathways of autophagy. Annu Rev Genet 43:67-93

25. Masiero E, Agatea L, Mammucari C et al (2009) Autophagy is required to maintain muscle mass. Cell Metab 10:507-515

26. Wu JJ, Quijano C, Chen E et al (2009) Mitochondrial dysfunction and oxidative stress mediate the physiological impairment induced by the disruption of autophagy. Aging (Albany NY) 1:425-437

27. Meijer AJ, Codogno P (2006) Signalling and autophagy regulation in health, aging and disease. Mol Asp Med 27:411-425

28. Lokireddy S, Wijesoma IW, Teng S et al (2012) The ubiquitin ligase mull induces mitophagy in skeletal muscle in response to musclewasting stimuli. Cell Metab 16:613-624 
29. Hojlund K, Frystyk J, Levin K, Flyvbjerg A, Wojtaszewski JF, Beck-Nielsen H (2006) Reduced plasma adiponectin concentrations may contribute to impaired insulin activation of glycogen synthase in skeletal muscle of patients with type 2 diabetes. Diabetologia 49:1283-1291

30. Vandesompele J, de Preter K, Pattyn F, et al (2002) Accurate normalization of real-time quantitative RT-PCR data by geometric averaging of multiple internal control genes. Genome Biol 3: RESEARCH0034

31. Hellemans J, Mortier G, de Paepe A, Speleman F, Vandesompele J (2007) qBase relative quantification framework and software for management and automated analysis of real-time quantitative PCR data. Genome Biol 8:R19

32. Mizushima N, Yoshimori T (2007) How to interpret LC3 immunoblotting. Autophagy 3:542-545

33. Moruno F, Perez-Jimenez E, Knecht E (2012) Regulation of autophagy by glucose in mammalian cells. Cells 1:372-395

34. Yan J, Feng Z, Liu J et al (2012) Enhanced autophagy plays a cardinal role in mitochondrial dysfunction in type 2 diabetic Goto-Kakizaki (GK) rats: ameliorating effects of (-)-epigallocatechin-3-gallate. J Nutr Biochem 23:716-724

35. Inami Y, Yamashina S, Izumi K et al (2011) Hepatic steatosis inhibits autophagic proteolysis via impairment of autophagosomal acidification and cathepsin expression. Biochem Biophys Res Commun 412:618-625

36. Yang L, Li P, Fu S, Calay ES, Hotamisligil GS (2010) Defective hepatic autophagy in obesity promotes ER stress and causes insulin resistance. Cell Metab 11:467-478

37. Codogno P, Meijer AJ (2010) Autophagy: a potential link between obesity and insulin resistance. Cell Metab 11:449-451

38. Koga H, Kaushik S, Cuervo AM (2010) Altered lipid content inhibits autophagic vesicular fusion. FASEB J 24:3052-3065

39. Mootha VK, Lindgren CM, Eriksson KF et al (2003) PGC-1alpharesponsive genes involved in oxidative phosphorylation are coordinately downregulated in human diabetes. Nat Genet 34: 267-273

40. Patti ME, Butte AJ, Crunkhorn S et al (2003) Coordinated reduction of genes of oxidative metabolism in humans with insulin resistance and diabetes: potential role of PGC1 and NRF1. Proc Natl Acad Sci U S A 100:8466-8471

41. Klionsky DJ, Abdalla FC, Abeliovich H et al (2012) Guidelines for the use and interpretation of assays for monitoring autophagy. Autophagy 8:445-554

42. Vendelbo MH, Moller AB, Christensen B et al (2014) Fasting increases human skeletal muscle net phenylalanine release and this is associated with decreased mTOR signaling. PLoS One 9, e102031

43. Mizushima N, Yamamoto A, Matsui M, Yoshimori T, Ohsumi Y (2004) In vivo analysis of autophagy in response to nutrient starvation using transgenic mice expressing a fluorescent autophagosome marker. Mol Biol Cell 15:1101-1111

44. Sala D, Ivanova S, Plana N et al (2014) Autophagy-regulating TP53INP2 mediates muscle wasting and is repressed in diabetes. J Clin Invest 124:1914-1927

45. Barazzoni R, Short KR, Asmann Y, Coenen-Schimke JM, Robinson MM, Nair KS (2012) Insulin fails to enhance mTOR phosphorylation, mitochondrial protein synthesis, and ATP production in human skeletal muscle without amino acid replacement. Am J Physiol Endocrinol Metab 303:E1117-E1125

46. Sancak Y, Bar-Peled L, Zoncu R, Markhard AL, Nada S, Sabatini DM (2010) Ragulator-Rag complex targets mTORC1 to the lysosomal surface and is necessary for its activation by amino acids. Cell 141:290-303

47. Efeyan A, Zoncu R, Chang S et al (2013) Regulation of mTORC1 by the Rag GTPases is necessary for neonatal autophagy and survival. Nature 493:679-683

48. Naito T, Kuma A, Mizushima N (2013) Differential contribution of insulin and amino acids to the mTORC1-autophagy pathway in the liver and muscle. J Biol Chem 288:21074-21081 